\title{
Preference for signaled shock phenomenon: Direct and indirect evidence for modifiability factors in the shuttlebox
}

\author{
JOHN J. FUREDY and GERALD B. BIEDERMAN \\ University of Toronto, Toronto, Ontario MSS IAI, Canada
}

\begin{abstract}
The experiments reported here investigated whether unauthorized shock modification is a factor which mediates the preference for signaled shock phenomenon (PSS) in the shuttlebox. This factor appeared not to have a critical role in PSS on the basis of earlier experiments which reported that PSS occurred in the shuttlebox even when shock was made unmodifiable by scrambling. However, the scrambling used in these experiments was not complete. In Experiments I and II reported here, no PSS occurred with completely scrambled grids; the phenomenon emerged only with unscrambled shock. Experiment III reports evidence indicating a connection between directly assessed modification and PSS with unscrambled shock, with PSS emerging only when modification had begun to occur. The pattern of results from these shuttlebox experiments parallels that found in an earlier study of PSS using a symmetrical-choice, barpress preparation.
\end{abstract}

The shuttlebox has provided the paradigmatic situation where the evidence for the theoretically significant preference for signaled shock phenomenon originated, (e.g., Lockard, 1963; Perkins, Seymann, Levis, \& Spencer, 1966) with time spent in the signaled shock compartment as the critical dependent measure of preference. The main significance of these reported PSS phemomena for a number of theoretical accounts of behavior was that PSS was found where the shocks were presumed by the experimenter to be both unavoidable and inescapable. The fact that signaling was still preferred despite the absence of response contingencies which might ameliorate the noxious event seems to provide strong support for interpretations that stress the importance of preparatory responses (e.g., Perkins, 1968), information per se (e.g., Berlyne, 1960), and safety (e.g., Seligman, 1968). On the other hand, as noted by Biederman and Furedy $(1970,1973)$, the theoretical significance of these shuttlebox experiments is potentially diminished by the consideration that the shuttlebox studies obtained the PSS phenomenon with shocks delivered through unscrambled foot grids, thus introducing and implicating modifiability factors as responsible for what had previously been interpreted as a preference for signaled, genuinely unavoidable, inescapable noxious events. This analysis received recent support in barpressing experiments (Biederman \& Furedy, 1970, 1973; Furedy \& Biederman, 1972). Experiment I reported by Biederman and Furedy (1973) is of closest relevance, where PSS (as measured by choosing a bar producing signaled shock rather than the other producing unsignaled shock) was obtained only

Experiments I, II, and III were supported by Canadian National Research Council grants and by an Atkinson Foundation grant to GBB and JJF, and by a Canada Council grant to JJF. We thank C. $X$. Poulos for his helpful comments on the manuscript, and G. A. Heighington for technical assistance.

Requests for reprints should be sent to J. J. Furedy, Department of Psychology, University of Toronto, Toronto, Ontario M5S 1A1, Canada. when the shocks were unscrambled, but not when they were unmodifiable as a function of scrambled foot grids or fixed tail electrodes. This indirect evidence for the role of modifiability in the PSS was supplemented by more direct evidence when it was also found that the degree of PSS in the unscrambled-shock group was correlated with the independently measured amount of unauthorized modification of signaled (unscrambled) shock (Biederman \& Furedy, 1973, Experiment II).

Modifiability, therefore, appears to play a crucial role in the PSS phenomenon in barpress studies involving a symmetrical ${ }^{1}$ choice between signaled and unsignaled shocks. On the other hand, no parallel evidence has been reported in the shuttlebox situation, despite the fact that studies based on this preparation are quoted in support of theoretical assertions such as the existence of preparatory responses (Perkins, 1968).

On the other hand, the evidence concerning the shuttlebox situation, on which most of the assertions supporting the notion of preparatory responses (e.g., Perkins, 1968) rests, is less clear. A conference report by Furedy and Walters $(1970)^{2}$ concluded that while modifiability did increase the PSS, in contrast to the barpress preparation, modifiability appeared not to be critical in producing the PSS phenomenon. Specifically, Furedy and Walters (1970) reported a significant, though weak, PSS effect even when the shock was apparently scrambled. However, reexamination by the present authors of the scrambled shock circuit for Furedy and Walters' (1970) report indicated that scrambling was only partial in that adjacent grid pairs had identical polarities, thus permitting possible unauthorized modification of shock. The first experiment, therefore, was a replication of the Furedy-Walters (1970) procedures, but the grid system was thoroughly checked so as to insure total scrambling in the condition designed to eliminate the modifiability factor. 


\section{EXPERIMENT I Manipulation of the scrambling factor}

\section{Method}

Subjects. 40 Wistar 90-day old male rats served as subjects.

Apparatus. Two shuttleboxes were used and constructed according to specifications given by Perkins, Levis, and Seymann (1963). Basically, this apparatus included a rectangular box with a removable center partition; preference for one compartment over the other was measured by the percent time spent on that side. The shock was $.6 \mathrm{~mA}$ delivered through footgrids. The grids delivered scrambled or unscrambled shock by means of a Grason-Stadler shock generator (Model E6070B). In the former case, positive and negative polarities alternated across the 18 grids; in the latter case the internal shock scrambler, consisting of a motor-driven commutator with 18 brushes, continuously varied the shock pattern across the 18 grids. The commutator turned at an approximate speed of $100 \mathrm{rpm}$ and its brushes were arranged so that a continuously changing shock pattern was produced. Thus, in contrast to the arrangement in Furedy and Walters (1970), the shock grids in the present experiment were thoroughly scrambled when appropriate. The signal consisted of the simultaneous onsets of both an $80-\mathrm{dB}$ tone (Mallory No. SC628) and ceiling $28-\mathrm{V}$ light bulb shielded so that it illuminated only the compartment occupied by the subject. The bulb was situated above the transparent Plexiglas ceiling. For all experiments, the boxes were housed in sound-attenuating insulated opaque chambers with one-way observation windows on top and a fan to maintain air circulation. All stimuli and interstimulus intervals were automatically programmed.

Procedure. On each testing day, the subject was "pretrained" as in Perkins et al. (1966), first by confinement for two $1 / 2-h$ periods in each of the two compartments, where the 5-sec duration shocks were delivered in a variable interval 120 -sec (ranging from 60 to $180 \mathrm{sec}$ ) response-independent schedule. The tone-light signal was $3 \mathrm{sec}$ in duration and the interval between tone-light and shock onsets was also $3 \mathrm{sec}$. In the "signaled" compartment, the tone-light signal preceded the shock onset in a 3-sec CS-US delay-conditioning paradigm; in the "unsignaled" side, shock onset preceded the tone-light signal onset by $3 \mathrm{sec}$. After two $1 / 2$-h pretraining sessions on the signaled and unsignaled sides (counterbalanced for order between subjects), the partition between the compartments was raised for a 3 -h period during which time the shock schedule was unchanged by the subject's behavior (i.e., intended to be inescapable and unavoidable shocks), but choice of side determined whether the shocks would be "signaled," "unsignaled," or (in the case of shuttling during the signal from signaled to unsignaled side) both "signaled" and "unsignaled." Thus, rats were pretrained for two $1 / 2-\mathrm{h}$ periods in the signaled and unsignaled sides for each of 2 days and then were allowed to freely choose between compartments for $3 \mathrm{~h}$ per day for 2 days. Half the subjects were run with scrambled shock, while the remaining 20 subjects were run with unscrambled shock.

\section{Results and Discussion}

The PSS phenomenon failed to emerge reliably when sources of unauthorized modification (cf. Furedy and Walters, 1970) were eliminated. The PSS emerged here as marginally significant in the first day only in the scrambled group $[\overline{\mathrm{X}}=64.8 \%, 16$ of 20 subjects above $50 \%, \mathrm{t}(19)=2.33, \mathrm{p}<.05]$. By the second day, even this marginal preference was reversed $\bar{X}=45.8 \%, 8$ of 20 subjects above $50 \%, \mathrm{t}<1]$. This failure to replicate Furedy and Walters' (1970) experiment insofar as the scrambled shock trend is concerned is consistent with the results reported by Biederman and Furedy (1970, 1973) and Furedy and Biederman (1972), but the failure to produce statistically reliable performance in the unscrambled shock condition was unexpected $(\overline{\mathrm{X}}$ Day $1=43.2 \%, 9$ of 20 subjects above $50 \%$; $\overline{\mathrm{X}}$ Day $2=54.5 \%, 11$ of 20 subjects above $50 \%$ ), especially considering the large sample size and other PSS rat studies with unscrambled shock in this laboratory (Biederman \& Furedy, 1970, 1973). Experiment II, therefore, was designed both to strengthen the unscrambled PSS phenomenon and, under more optimal conditions, to check again on the question of whether scrambling would not only reduce but perhaps actually eliminate the PSS phenomenon.

\section{EXPERIMENT II \\ Manipulation of the scrambling factor without pretraining}

The practice of pretraining or preexposure to the signaled and unsignaled conditions has been standard for all shuttlebox experiments since the first reported by Lockard (1963). The adoption of this practice in our experiments was based both on a desire to replicate previous conditions as well as the notion that it was advisable to provide this form of "indication" to the subjects of the two states between which they had to choose. However, continuous observation of the subjects run in Experiment I indicated that animals confined to one side or the other during pretraining would often freeze. It was therefore considered possible that behavioral freezing might interfere with the registration of preference to a degree that would outweigh the "beneficial" effects arising from the indicator function of the pretraining-confinement periods. It was further suspected that such freezing specifically retarded the performance in unscrambled conditions.

\section{Method}

The number and type of subjects, apparatus, and procedure exactly paralleled Experiment I, except that on the two test days all subjects were simply given the $3 \mathrm{~h}$ of free-choice time without any preceding periods of confinement in the two sides.

\section{Results and Discussion}

The time spent in the signaled side by the unscrambled group was not only greater in this experiment than in Experiment 1 , but the PSS phenomenon emerged as significant not only by Day $2[\overline{\mathrm{X}}=73.0 \%, 17$ of 20 subjects above $50 \%, \mathrm{t}(19)=3.28, \mathrm{p}<.01]$, but even on Day $1[\bar{X}=63.4 \%, 14$ of 20 subjects above $50 \%$, $t(19)=2.20, p<.05]$. On the other hand, as in Experiment I, no significant PSS emerged in the scrambled group of subjects on Day 2 of testing $(\bar{X}=59.7 \%$, 14 of 20 subjects above $50 \%, t=1.12$ ); in Experiment II Day 1 also produced no PSS [ $\overline{\mathrm{X}}=46.5 \%, 9$ of 20 subjects above $50 \%, \mathrm{t}(19)<1]$. These findings are con- 
sistent with a view that in an optimal situation where preference for signal emerges when shock is modifiable, scrambling eliminates the preference on both Day 1 and Day 2 of training. These results would indicate that the Furedy-Walters (1970) results have failed in replication and do not warrant citation as evidence for (scrambled) PSS (e.g., Cantor \& LoLordo, 1972). The present experiment further suggests that elimination of pretraining strengthens the PSS phenomenon with modifiable (unscrambled) shocks.

To this point, however, the modifiability factor's role in the PSS phenomenon has only been indirectly examined. The final experiment measured modification itself in the unscrambled-shock condition in an arrangement which yielded the strongest PSS effect, i.e., the no-pretraining unscrambled arrangement of the present experiment.

\section{EXPERIMENT III}

\section{Directly examined modification and its relation to PSS}

\section{Method}

20 subjects served; apparatus and procedure were identical with the no-pretraining unscrambled group in Experiment II. The amount of unauthorized modification of the shock was evaluated by means of a recording milliammeter in series with the subject, as in Biederman and Furedy (1973, Experiment I).

\section{Results and Discussion}

In the present experiment, a preference for signaled shock was found comparable to that in the corresponding group in Experiment II. The mean preference was $73.7 \%$ on Day $1(\mathrm{p}<.01)$ and $72.0 \%$ on Day 2 $(\mathrm{p}<.05)$. This reliable preference confirms the optimal strategy used in Experiment II to promote preference for signaled unscrambled shock. Shock modification was assessed by visual inspection of the record of the milliammeter; an unmodified shock circumscribed an area of 10 rectangles on the recorder paper while a completely modified (avoided) shock would only slightly displace the pen (about 3 rectangles would remain, because of current absorbed in the recording circuit); modifications were noted by simply counting the number of rectangles under each shock curve.

The sort of milliammeter output used to obtain direct evidence for shock modification is shown in Figure 1. As the bottom panel (scrambled shock) indicates, all unmodified shocks produced essentially the same wave forn, with area under the curve directly related to amount of shock received. However, with unscrambled shock (top panel in Figure 1) used in the present experiment, there are readily detectable changes in wave form and a corresponding reduction in area under the curve(i.e., Trials 2, 3, 4, 6, 7, and 9 in Figure 1); there is no appreciable difference in wave-form between unmodified shocks in unscrambled or scrambled conditions. It should also be noted that there is no characteristic pattern of modification, when it did occur (contrast Shock 3 with Shock 6 in top panel for respective examples of early and late shock modification).

We observed only very infrequent cases of total modification (3 rectangles remaining); the infrequency of occurrence of total modification confirms that reported by Lockard (1963), who only measured this effect on a two-point scale (present or absent), and found it unrelated to PSS. The milliammeter recordings of the sort shown in Figure 1 allowed modification to be measured more continuously in the present experiment. Specifically, a grid system was used to estimate area under the curve, and generated an 8-point scale of percent modification (\%M), ranging from 100 to 0 in 12.5-point steps.

Inspection of trial-by-trial $\% \mathrm{M}$ values for each subject revealed a clear step function (increase from negligible to appreciable \%M values) occurring over trials in 12 subjects during Day 1 and in 10 subjects during Day 2 performance. These data are summarized in the upper panel of Figure 2. For each subject the demarcation point or criterion trial (C) was a trial prior to which no substantial modification occurred, whereas after the $C$ trial, as shown in Figure 2 (upper panel), there was substantial modification. For the remaining 8 and 10 subjects during Day 1 and Day 2 performance, respec-

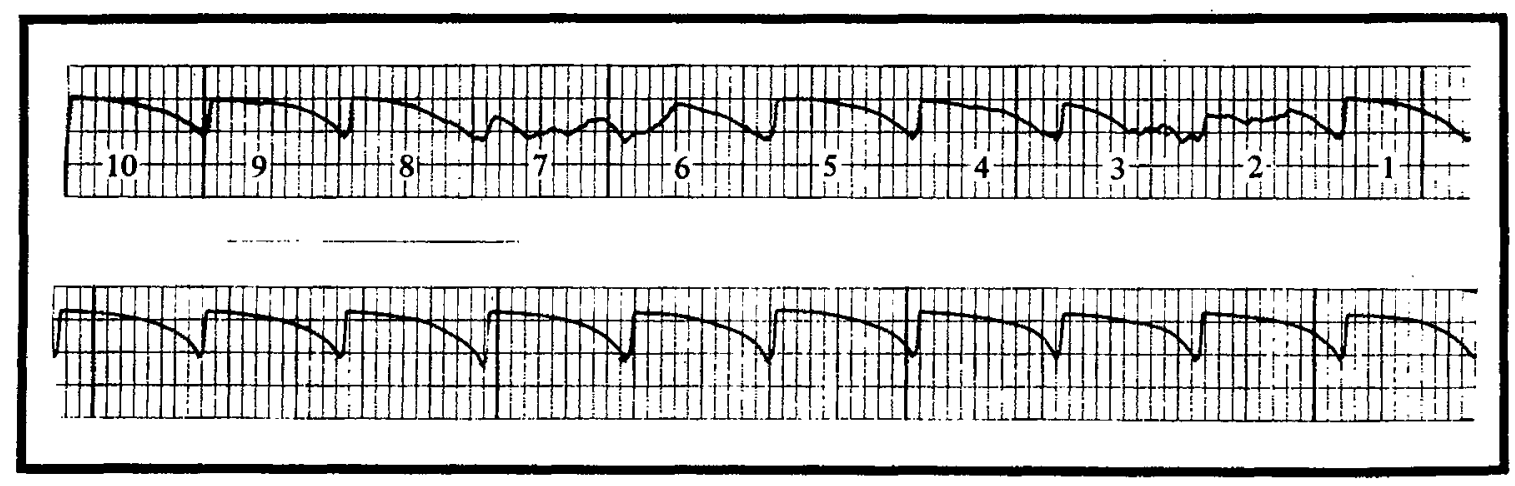

Figure 1. Segment of milliammeter records for a scrambled shock subject (bottom panel) and an unscrambled shock subject (top panel). Time (one small division per $1 / 2$-sec) flow is right to left. One large vertical division represents approximately $0.5 \mathrm{~mA}$. 

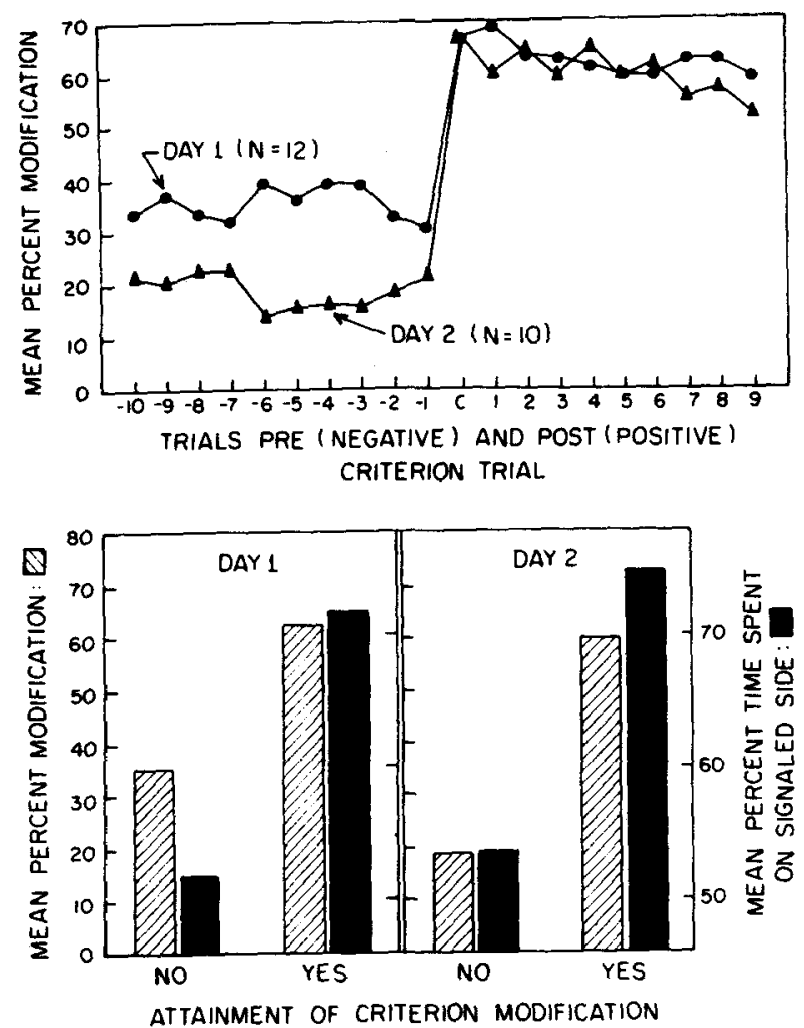

Figure 2. Trial-by-trial modification performance of subjects manifesting the step function (top panel), and the relationship between the attainment of modification and emergence of preference for signaled side (bottom panel).

tively, substantial modification existed virtually from the first trial. In these subjects the first trial satisfied the definition of the $C$ trial for the step-function subjects (shown in Figure 2, upper panel), which was that $C$ was the earliest trial which began a series of 10 trials whose mean \%M was equal to the mean \%M on the last block of 10 trials $( \pm 5 \%)$ of that day.

The fact that an appreciable proportion of subjects showed a step function (attainment of modification at a clearly definable demarcation point) meant that the relationship between the development of modification and of the PSS phenomenon could be examined on an individual-subject, within-trials basis. The trends resulting from that examination are summarized in the lower panel of Figure 2, which shows both \%M and the PSS (percent time spent on signaled side) as a function of whether subjects had not ("no") or had ("yes") attained the criterion of appreciable modification. Precriterion PSS during Day 1 was based only on 8 of the 12 subjects showing the stepwise \%M scores, because the remaining 4 subjects spent $100 \%$ of their time in either the signaled or the unsignaled side. Since these subjects had had no experience with both sides, a "preference" did not legitimately exist, so that their scores could not be used. However, by Day 2, all subjects had spent some time in both signaled and unsignaled sides, so that all 10 subjects showing the stepwise \%M scores on that day could be used for assessing precriterion PSS.

These statistical assessments, as suggested by the trends in Figure 2 (lower panel), indicated no significant precriterion PSS, $[\mathrm{t}(7)=1.03$ and $\mathrm{t}(9)=0.40$ for Days 1 and 2, respectively, ps $>.05]$. However, postcriterion preference was significant $[p<.01$ for both Day 1 and Day 2, $t(19)=3.72$ and 3.49, respectively] It should also be noted that, as suggested by the trends in both panels of Figure 2, there were no systematic day-to-day differences with respect to amount of PSS, amount of modification, or even number of subjects showing the stepwise development of modification over trials for a given day. In other words, no day-today "learning" seemed to have occurred either of the PSS or of modification, although the within-day development of each was obviously related, inasmuch as premodification subjects showed no preference for signal, whereas subjects extensively modifying shock showed a significant preference for signal.

\section{GENERAL DISCUSSION}

It would appear that the results reported by Furedy and Walters (1970) should not be used as evidence (cf. Cantor \& LoLordo, 1972) for preference for signaled unmodifiable shock inasmuch as these experiments failed to show preference in a reliable scrambled shock procedure. Experiment I showed no significant preference by Day 2 when rigorous scrambling procedures were used. In one group a marginal PSS was found with scrambling on Day 1 (64.8\%), but this fell away on Day 2 with the group PSS dropping to $45.8 \%$. The evidence in Biederman \& Furedy (1973), which indicated no preference for unmodifiable signaled shock in a symmetrical-choice ${ }^{1}$, barpress preference situation has now been confirmed in the shuttlebox paradigm. When the usual pretraining strategy was removed in an unscrambled group (Experiment III), a clear preference for signal emerged which was found to be related to modification of the shock; this shuttlebox evidence is consistent with the earlier barpress study (Biederman \& Furedy, 1973), where unauthorized modification of shock assessed in the same direct way was also found to be connected with the amount of independently assessed PSS. However, in that experiment the modification-PSS connection could only be asserted on the basis of a correlation between the extents of the two factors. Since a sizeable proportion of subjects in the present shuttlebox experiment (III) exhibited a clear stepwise function from negligible to appreciable modification over trials, it was possible to more closely relate the development of modification with that of the PSS.

The direct, milliammeter-recording assessment of modification obtained in Experiment III at least suggests that the shock modification involved in the PSS phenomenon is not of the more internal sert suggested by such conceptualizations as "preception" (e.g., Lykken, 
1962). Rather, at least in the classic shuttlebox preparation used to assess the PSS (e.g., Lockard, 1963), the present results suggest that PSS emerges when animals develop the capacity for external and unauthorized shock modification by spending an appreciable time on grids of the same polarity during the delivery of the supposedly inescapable, but unscrambled, shock.

\section{REFERENCES}

Badia, P., Coker, C. C., \& Harsh, J. Choice of higher density signaled shock over lower density unsignaled shock. Journal of the Experimental Analysis of Behavior, 1973, 20, 47-55.

Badia, $P$, \& Culbertson, $S$. The relative aversiveness of signaled vs. unsignaled escapable and inescapable shock. Journal of the Experimental Analysis of Behavior, 1972, 17, 463-471.

Badia, P., Culbertson, S., \& Harsh, J. Choice of longer or stronger signaled shock over shorter or weaker unsignaled shock. Journal of the Experimental Analysis of Behavior. $1973,19,25-32$.

Berlyne, D. E. Conflict, arousal, and curiosity. New York: McGraw-Hill, 1960.

Biederman, G. B., \& Furedy, J. J. The preference-for-signaledshock phenomenon: signaling shock is reinforcing only if shock is modifiable. Quarterly Journal of Experimental Psychology, 1970, 22, 681-685.

Biederman, G. B., \& Furedy, J. J. Preference-for-signaled-shock phenomenon: effect of shock modifiability and light reinforcement. Journal of Experimental Psychology, 1973, 100, 380-386.

Cantor, M. B., \& LoLordo, V. M. Reward value of brain stimulation is inversely related to uncertainty about its onset. Journal of Comparative and Physiological Psychology, 1972, 79, 259-270.

Furedy, J. J., \& Biederman, G. B. Development of the reinforcing effect of signaling modifiable shock. Perceptual and Motor Skills, 1972, 35, 31-34

Furedy, J.J., \& Biederman, G. B. Methodological problems in evaluating rat preference for signaled or unsignaled shock. Paper presented to Psychonomic Society, 1974.
Furedy, J. J., \& Walters, G. C. Preference for signaled, supposedly-unmodifiable shock as a function of scrambling the grid. Paper presented to Psychonomic Society, 1970.

Lykken, D. T. Preception in the rat: autonomic response to shock as a function of length of warning interval. Science, $1962,137,665-666$.

Lockard, J. S. Choice of a warning signal or no warning signal in an unavoidable shock situation. Journal of Comparative and an unavoidable shock situation. Journal of C
Physiological Psychology, 1963, 56, 526-530.

Perkins, C. C., Jr. An analysis of the concept of reinforcement. Psychological Review, 1968, 75, 155-172.

Perkins, C. C., Jr., Levis, D. J., \& Seymann, R. G. Preference for signal-shock vs. shock-signal. Psychological Reports, 1963, 13, 735-738.

Perkins, C. C., Jr., Seymann, R. G., Levis, D. J., \& Spencer, H., Jr. Factors affecting preference for signal-shock over shocksignal. Journal of Experimental Psychology, 1966, 72. 190-196.

Seligman, $\dot{M}$. E. P. Chronic fear produced by unpredictable electric shock. Journal of Comparative and Physiological Psychology, 1968, 66, 402-411.

\section{NOTES}

1. As discussed in more detail elsewhere (Furedy \& Biederman, 1974), the apparently strong evidence for PSS with unmodifiable shock reported by Badia and his associates (Badia, Coker, \& Harsh, 1973; Badia \& Culbertson, 1972; Badia, Culbertson, \& Harsh, 1973) using a "changeover" paradigm presents the animal with an asymmetrical choice, inasmuch as an active (barpress) response produces signal whereas a different, passive (withholding-of-barpress) response produces unsignaled shock. In addition to methodological problems arising from this asymmetry, there is evidence that this form of changeover is operationally, but not behaviorally, replicable and that there may, after all, be even technical problems which permit shock modification to occur (Furedy \& Biederman, 1974, pp. 9-10) in the apparatus used by Badia and associates.

2. A copy of this paper is available on request to J. J. Furedy.

(Received for publication November 26, 1974. Revision accepted June 18,1975 .) 